\title{
Probabilistic Financial Community Models with Latent Dirichlet Allocation for Financial Supply Chains
}

\author{
Zheng Xu \\ University of Maryland \\ xuzh@cs.umd.edu
}

\author{
Louiga Raschid \\ University of Maryland \\ louiqa@umiacs.umd.edu
}

\begin{abstract}
There is a growing interest in modeling and predicting the behavior of financial systems and supply chains. In this paper, we focus on the the analysis of the resMBS supply chain; it is associated with the US residential mortgage backed securities and subprime mortgages that were critical in the 2008 US financial crisis. We develop models based on financial institutions (FI), and their participation described by their roles (Role) on financial contracts (FC). Our model$\mathrm{s}$ are based on an intuitive assumption that FIs will form communities within an FC, and FIs within a community are more likely to collaborate with other FIs in that community, and play the same role, in another FC. Inspired by the Latent Dirichlet Allocation (LDA) and topic models, we develop two probabilistic financial community models. In FIComm, each FC (document) is a mix of topics where a topic is a distribution over FIs (words). In Role-FI-Comm, each topic is a distribution over Role-FI pairs (words). Experimental results over $5000+$ financial prospecti demonstrate the effectiveness of our models.
\end{abstract}

\section{Keywords}

Latent Dirichlet allocation; generative probabilistic model; financial supply chain; mortgage backed securities.

\section{INTRODUCTION}

There is growing interest in modeling and predicting the behavior of financial systems and supply chains. With the success of text extraction tools, and the availability of public financial documents that are typically filed with the Securities and Exchange Commission (SEC), there is an opportunity to create financial big data collections. Some recent examples include text extraction from financial prospecti and company annual reports $[2,3,4,7]$. In this paper, we study the supply chain for US residential mortgage backed securities, resMBS [2]. This system combined with the subprime mortgage crisis to lead to the 2008 U.S. financial crisis. Our

Permission to make digital or hard copies of all or part of this work for personal or classroom use is granted without fee provided that copies are not made or distributed for profit or commercial advantage and that copies bear this notice and the full citation on the first page. Copyrights for components of this work owned by others than ACM must be honored. Abstracting with credit is permitted. To copy otherwise, or republish, to post on servers or to redistribute to lists, requires prior specific permission and/or a fee. Request permissions from permissions@ acm.org.

DSMM'16, June 26-July 01 2016, San Francisco, CA, USA

(c) 2016 ACM. ISBN 978-1-4503-4407-4/16/06 . . $\$ 15.00$

DOI: http://dx.doi.org/10.1145/2951894.2951900 interest is in the the complex financial networks that describe this supply chain, i.e., the financial institutions (FI) and their role(s) in financial contracts (FC).

Figure 1 illustrates the summary section of a prospectus for a mortgage backed security. Text extraction will enable us to extract the names of financial institutions (FI names). Example FI names in this summary are Wachovia Bank, National City, HSBC Bank, etc. We can also extract the Role played by the FIs, e.g., depositor, issuing entity, seller, sponsor, originator, servicer, trustee, etc. The lower part of the figure illustrates the output of text extraction of FI names and Role-FI matching from the summary section. For example, we note that Wachovia, identified as FI380, plays the role of depositor, issuing entity, seller and sponsor, for this exemplar FC. Similarly, National City Bank, identified as FI263, plays the role of originator and servicer.

Our intuitive assumption is that FIs will naturally form communities within an FC, and that FIs within a community are more likely to collaborate with other FIs in that community, and play the same role, in another FC. We adapt the Latent Dirichlet Allocation (LDA) [1] that were applied to collections of documents to this domain.

We develop two probabilistic financial community models, FI-Comm and Role-FI-Comm. FI-Comm captures the co-occurrence of FIs within an FC and Role-FI-Comm captures the co-occurrence of Role-FI pairs within an FC. In FI-Comm, we treat each FC (document) as a bag of FIs (words), and represent each FC as a random mix over latent FI communities (topics), where each community is characterized by a distribution over FIs. In Role-FI-Comm, we treat each FC (document) as a bag of Role-FI pairs (words), and represent each FC as a random mix over latent Role-FI communities (topics), where each community is characterized by a distribution over Role-FI pairs. The discovered FI or Role-FI probabilistic communities can be used to explain the behavior of FIs within an FC or to predict FIs and the role they played in FCs.

To our knowledge, we are the first to apply probabilistic graphical models to understand the behavior of financial supply chains. Comparing with the clustering approach for communities discussed in [2], our probabilistic model assign FIs into communities with some underlying assumption of probability distribution. FIs in our probabilistic communities are aligned with weights. Moreover, our probabilistic community could easily incorporate roles, and has the potential to be naturally extended to include structural and temporal information.

We present preliminary experimental results for the FI- 


\begin{tabular}{|c|c|c|}
\hline \multicolumn{3}{|c|}{ Summary section of WACHOVIA_1393388_0001068238-07-000417 } \\
\hline \multicolumn{3}{|c|}{$\begin{array}{l}\text { Title of Series............................ Wachovia Mortgage Loan Trust, LLC Mortgage Pass-Through } \\
\text { Certificates, Series 2087-A. }\end{array}$} \\
\hline \multicolumn{3}{|c|}{ 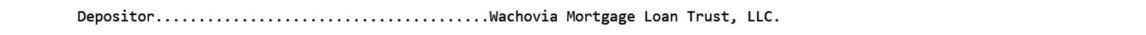 } \\
\hline \multicolumn{3}{|c|}{ 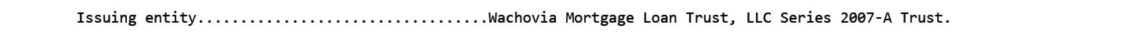 } \\
\hline \multicolumn{3}{|c|}{ Seller and Sponsor..........................Wachovia Bank, National Association. } \\
\hline \multicolumn{3}{|c|}{ 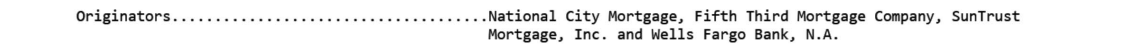 } \\
\hline \multicolumn{3}{|c|}{ 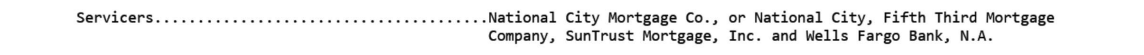 } \\
\hline \multicolumn{3}{|c|}{ Trustee $\ldots \ldots \ldots \ldots \ldots \ldots \ldots \ldots \ldots \ldots \ldots \ldots \ldots \ldots \ldots \ldots \ldots \ldots \ldots \ldots$ HSBC Bank USA, National Association. } \\
\hline \multicolumn{3}{|c|}{$\begin{array}{l}\text { Master Servicer and } \\
\text { Certificate Administrator.....................s. Bank National Association. }\end{array}$} \\
\hline \multicolumn{3}{|c|}{ Extracted tuples: } \\
\hline $\begin{array}{l}\text { (Role; } \\
\text { (Depositor; } \\
\text { (Issuing Entity; } \\
\text { (Seller; } \\
\text { (Sponsor; } \\
\text { (Originators; } \\
\text { (Originators; } \\
\text { (Originators; } \\
\text { (Servicers; } \\
\text { (Servicers; } \\
\text { (Servicers; } \\
\text { (Trustee; } \\
\text { (Master Servicer; }\end{array}$ & $\begin{array}{l}\text { Dict-NER Participant; } \\
\text { Wachovia Mortgage Loan Trust, LLC; } \\
\text { Wachovia Mortgage Loan Trust, LLC Series 2007-A; } \\
\text { Wachovia Bank, National Association; } \\
\text { Wachovia Bank, National Association; } \\
\text { National City Mortgage; } \\
\text { Fifth Third Mortgage Company; } \\
\text { SunTrust Mortgage, Inc.; } \\
\text { National City Mortgage Co.; } \\
\text { National City; } \\
\text { Fifth Third Mortgage Company; } \\
\text { HSBC Bank Association; } \\
\text { U.S. Bank National Association; }\end{array}$ & $\begin{array}{l}\text { Rank-ER FI-ID) } \\
\text { FI380:Wachovia), } \\
\text { FI380:Wachovia), } \\
\text { FI380:Wachovia), } \\
\text { FI380:Wachovia), } \\
\text { FI263:National City), } \\
\text { FI145:Fifth Third Bank), } \\
\text { FI345:SunTrust), } \\
\text { FI263:National City), } \\
\text { FI263:National City), } \\
\text { FI145:Fifth Third Bank) } \\
\text { FI183:HSBC), } \\
\text { FI363:U.S. Bank), }\end{array}$ \\
\hline
\end{tabular}

Figure 1: Summary section of an example prospectus and the extracted 3-tuples.

Comm and Role-FI-Comm models. Our dataset is the extracted resMBS dataset from $5000+$ prospecti for mortgage backed securities that were filed with the SEC between 2002 and 2008. We evaluate the quality of the discovered FI and Role-FI communities (topics) using a coherence metric. We evaluate the explanation and generation ability of our models using a perplexity metric. We also perform a qualitative evaluation by examples.

Our results reflect that both FI-Comm and Role-FI-Comm topics capture important knowledge about this supply chain. Of particular interest is that during the financial crisis, when bankruptcies may have disrupted communities, results from Role-FI-Comm indicate that surviving companies continued to play the same role in their communities.

\section{DATASET CREATION}

Figure 2 shows the pipeline to extract financial institutions (FI) who are participants in a financial contract (FC), and their roles in the FC. The extraction pipeline is comprised of several components. A pre-processing module detects the header and summary sections; it must be robust to handle a range of document format and layout issues.

Dict NER [9] is a special purpose Named Entity Recognizer that is tuned to extract FI names. FI names are composed of a root, which is usually unique, and a suffix which is drawn from a small corpus of suffix terms. Dict NER utilizes both a root dictionary and a suffix dictionary to recognize FI names. The Role Extraction module uses keyword matching to extract roles such as issuer, depositor, sponsor, etc. This is followed by a Role Participant matching unit which pairs a role with one or more FI names. Rank ER [9] performs entity resolution on the extracted FI name and maps each FI name to a corpus of standardized FI names obtained from the ABSNet portal. The standardized FI from ABSNet often contains the representative financial name for a financial family, which causes RankER to merge a financial family to the same FI. Differentiate subsidiaries will slightly change the results, but it is out of the scope of this paper.

The extraction pipeline is developed using the rule-based algebraic information extraction system, SystemT [5]. The advantages of a rule-based extraction approach has been demonstrated in [6]. One benefit is the ability to provide an explanation of successes and errors.

The upper section of Figure 1 shows the summary section of an exemplar prospectus while the lower section shows a 3-tuple corresponding to each Role-FI pair. The elements of each 3-tuple include the role that an FI plays in the FC, the FI name mention extracted by Dict-NER, and the standardized FI name (FI-ID) that is computed by Rank ER.

We also extract the date the FC was issued as well as the nominal $\$$ value of the FC. Details of Dict NER and Rank ER are in [9] and details of the extraction process is in [2].

\section{FINANCIAL COMMUNITY MODEL}

Our model is informed by the Latent Dirichlet Allocation (LDA) and its application to develop probabilistic topic models for text corpora. We apply a similar reasoning to identify two probabilistic financial community models. In the description of the model, we use FC to denote a financial contract, FI to denote a financial institution, and Role-FI to denote a pair comprising a role and an FI.

LDA [1] is a generative probabilistic model for collections of discrete data (documents) as shown in Figure 3. For a collection of $\mathrm{M}$ documents, LDA represents each document as a random mix over latent topics. Each topic is characterized by a distribution over words. Given the hyper-parameters $\alpha$ and $\beta$, the probability of a document with $\mathrm{N}$ words is

$$
p(\mathbf{w} \mid \alpha, \beta)=\int p(\theta \mid \alpha)\left(\prod_{n=1}^{N} \sum_{z_{n}} p\left(z_{n} \mid \theta\right) p\left(w_{n} \mid z_{n}, \beta\right)\right) d \theta
$$

where $\mathbf{w}=\left\{w_{1}, w_{2}, \ldots, w_{N}\right\}$ is a set of $N$ words, $\theta$ is a topic mixture sampled from a Dirichlet distribution param- 


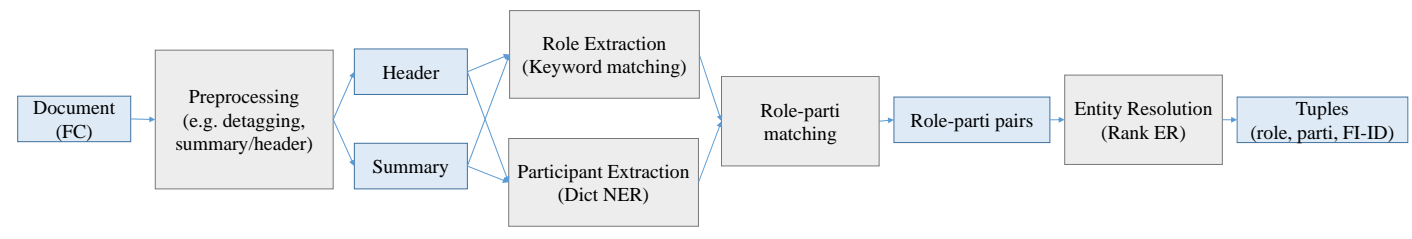

Figure 2: The extraction pipeline. The input is the financial contract (FC), and the outputs are a set of 3-tuples. Each 3-tuple is comprised of three elements, role, participant and FI as in Figure 1.

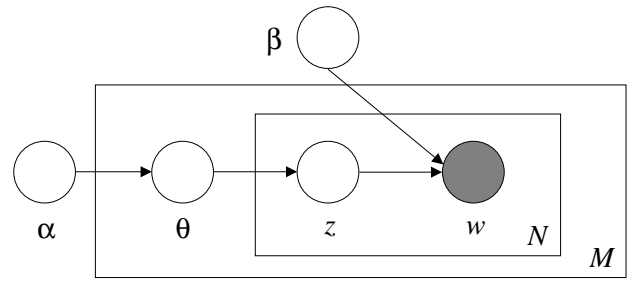

Figure 3: A graphical representation of LDA for a text corpus [1]. The boxes are "plates" representing replicates, where the outer plate represent $M$ documents, the inner plate represents the repeated choice of topics and words within a document.

eterized by $\alpha, z_{n}$ represents a topic sampled from a multinomial distribution conditioned on $\theta$, and each word $w_{n}$ is sampled from a multinomial distribution conditioned on $z_{n}$ and parameterized by $\beta$.

LDA is a three-level hierarchical Bayesian model. The parameters $\alpha$ and $\beta$ are corpus level parameters, assumed to be sampled once in the process of generating a corpus. The variables $\theta$ are document-level variables, sampled once per document. Finally, the variables $z_{n}$ and $w_{n}$ are word-level variables, sampled once for each word in each document.

Informed by LDA topic models, we build two models, FI-Comm and Role-FI-Comm. FI-Comm captures the cooccurrence of FIs within an FC and Role-FI-Comm captures the co-occurrence of Role-FI pairs within an FC. In FI-Comm, we treat each FC (document) as a bag of FIs (words), and represent each $\mathrm{FC}$ as a random mix over latent probabilistic FI communities, where each probabilistic FI community is characterized by a distribution over FIs. In Role-FI-Comm, we treat each FC (document) as a bag of Role-FI pairs (words), and represent each FC as a random mix over latent probabilistic Role-FI communities, where each probabilistic Role-FI community is characterized by a distribution over Role-FI pairs.

\section{EXPERIMENTAL RESULTS}

In this section, we discuss our empirical evaluation of FI-Comm and Role-FI-Comm. We start by describing the dataset. Then the FI-Comm and Role-FI-Comm are quantitatively evaluated by the perplexity metric and qualitatively evaluated by exemplar FCs. Moreover,, we validate the communities using a coherence metric.

\subsection{Experimental setup}

We extracted tuples as discussed in Section 2 from a collection of $5000+$ resMBS prospecti that were filed with the
SEC between 2002 and 2008. For both models, we filtered out FIs that appear in less than 20 FCs and FCs that contain less than 3 FIs. For FI-Comm, the resulting dataset included 3146 FCs, a vocabulary of 96 FIs and (a word count of) 36945 FIs. For Role-FI-Comm, the dataset included 4472 FCs, a vocabulary of 267 Role-FIs and 41075 Role-FIs. We implement FI-Comm and Role-FI-Comm using the Python sklearn toolkit $^{1}$

We used the natural split of the dataset based on the year of issue of the contract. This is aligned with our intuition that an FI would participate in a community in an FC, and then continue to participate in that community in a future FC. We used FCs from 2002 to 2005 to fit the models. We evaluated the data on the seen FCs and the unseen FCs from 2006 to 2008. We also created a synthetic dataset of FCs, where the yearly count of FCs and the count of words in each FC matched the real dataset. For each document in the synthetic dataset, we used the histogram representing the probability distribution of FIs and Role-FI pairs, for that year, to create the bag of words. We compare the performance of the models on the real and synthetic dataset using the normalized perplexity metric.

\subsection{Model Performance}

We use the perplexity metric for the FC dataset in each year to determine the generalizability of the models and the ability to fit the seen data (2002 to 2005) and to predict the unseen data (2006 to 2008). Perplexity is commonly used to evaluate language models; the score will monotonically decrease with the average likelihood of words. A lower perplexity score indicates better generalization potential. For a test set $D_{\text {test }}$ of $M$ documents, where $N_{d}$ is the number of words in the $d$-th document and where $p\left(\mathbf{w}_{d}\right)$ is the likelihood of document $\mathbf{w}_{d}$ for the given model, the perplexity score is as follows:

$$
\operatorname{Perp}\left(D_{\text {test }}\right)=\exp \left(-\frac{\sum_{d=1}^{M} \log p\left(\mathbf{w}_{d}\right)}{\sum_{d=1}^{M} N_{d}}\right)
$$

The normalized perplexity for each year is the perplexity for the FCs in the real dataset for that year, $D_{\text {real }}(y e a r$ ) divided by the perplexity for the FCs in the synthetic dataset for that year, $D_{\text {synth }}($ year $)$, as follows:

$$
N o r m P e r p(y e a r)=\frac{\operatorname{Perp}\left(D_{\text {real }}(\text { year })\right)}{\operatorname{Perp}\left(D_{\text {synth }}(\text { year })\right)} \text {. }
$$

Figure 5 shows the perplexity and the normalized perplexity. Each line represents the scores for seen data (2002 to 2005) and unseen data (2006 to 2008). The $\mathrm{X}$ axis is the number of probabilistic communities. Figures 5(a) and (b) correspond

\footnotetext{
${ }^{1}$ http://scikit-learn.org/stable/modules/generated/sklearn. decomposition.LatentDirichletAllocation.html
} 
Probabilistic communities discovered by LDA in Role-FI-Comm

\begin{tabular}{|c|c|c|c|}
\hline $\begin{array}{l}\text { masterservicer+FI384(Wells Fargo): } \\
\text { servicer+FI46(Bank of America): } \\
\text { seller+FI46(Bank of America): } \\
\text { depositor+FI44(Banc of America Securities LLC): } \\
\text { seller+FI106(DLJ Mortgage Capital Inc.): }\end{array}$ & $\begin{array}{l}0.0614 \\
0.0605 \\
0.0578 \\
0.0460 \\
0.0373\end{array}$ & $\begin{array}{l}\text { depositor+FI102(Countrywide Securities Corporation): } \\
\text { masterservicer+FI101(Countrywide Home Loans): } \\
\text { seller+FI101(Countrywide Home Loans): } \\
\text { seller+FI384(Wells Fargo): } \\
\text { trustee+FI47(Bank of New York Mellon Corp.): }\end{array}$ & $\begin{array}{l}0.1682 \\
0.1123 \\
0.0882 \\
0.0491 \\
0.0447\end{array}$ \\
\hline $\begin{array}{l}\text { masterservicer+FI384(Wells Fargo) } \\
\text { securitiesadministrator+FI384(Wells Fargo) } \\
\text { depositor+FI50(Bear Stearns \& Co. Inc.) } \\
\text { seller+FI119(EMC Mortgage) } \\
\text { issuer+FI50(Bear Stearns \& Co. Inc.) }\end{array}$ & $\begin{array}{l}0.1362 \\
0.0863 \\
0.0645 \\
0.0481 \\
0.0372\end{array}$ & $\begin{array}{l}\text { masterservicer+FI34(Aurora Loan Services LLC) } \\
\text { depositor }+ \text { FI344(Structured Asset) } \\
\text { seller+FI223(Lehman Brothers) } \\
\text { servicer+FI34(Aurora Loan Services LLC) } \\
\text { sponsor +FI223(Lehman Brothers) }\end{array}$ & $\begin{array}{l}0.1202 \\
0.1056 \\
0.0691 \\
0.0363 \\
0.0325\end{array}$ \\
\hline \multicolumn{4}{|c|}{ Example FC with most likely community assignments } \\
\hline FC4415 SASCO_1332166_0001125282-05-0035 & & Year 2005 & \\
\hline
\end{tabular}

servicer+FI291; servicer+FI285; servicer+F1384; masterservicer+FI34; masterservicer+FI344; originator+FI291; originator+FI146; issuin

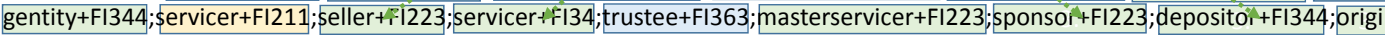
nator+FI34; seller+FI344; sponsor+FI344; depositor+FI223

\begin{tabular}{|l|l|l}
\hline FC993 CITIGROUP_1407876_0000882377-07-001984.htm & Year 2007 & $0.8009 ; 0.0077 ; 0.0625 ; 0.1289$ \\
\hline
\end{tabular}

trustadministrator+FI82; trustadministrator+FI84; trustadministrator+FI85; servicer+FI384; servicer+ FI180; depositor+FI84; sponsor+FI84; originator+FI180; issuingentity+FI84; servicer+FI102; originator+ FI101; trustee+FI85; servicer+FI101; originator+FI384; trustee+FI363; masterservicer+FI84; masterservi cer+FI82; seller+FI84; trustee+FI384

Figure 4: Financial communities (topics) from Role-FI-Comm. The top section shows the Top 5 Role-FIs for four communities. The lower section shows two financial contracts (FC); color coding connects the Role-FIs in the FCs to the topics. The figure is best viewed in color.

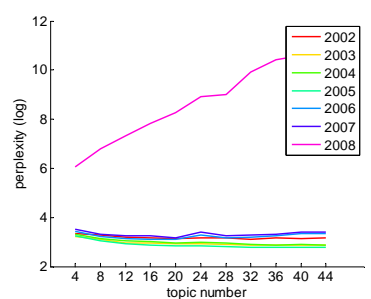

(a)

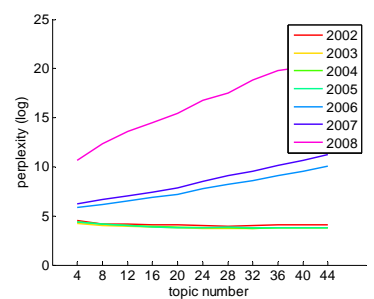

(c)

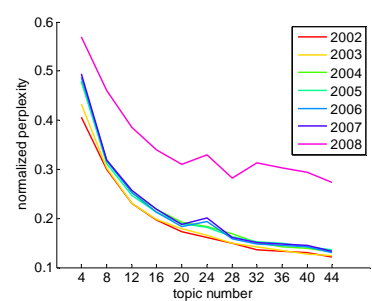

(b)

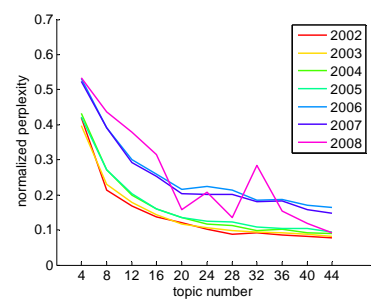

(d)
Figure 5: Perplexity and normalized perplexity for FCs in each year. Each line represents the score for seen data (2002 to 2005) and unseen test data (2006 to 2008). The $X$ axis is the number of probabilistic communities. (a) and (b) correspond to FI-Comm and (c) and (d) to Role-FI-Comm. The figure is best viewed in color.

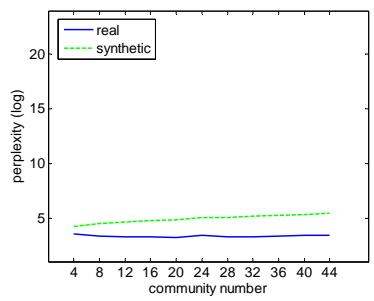

(a)

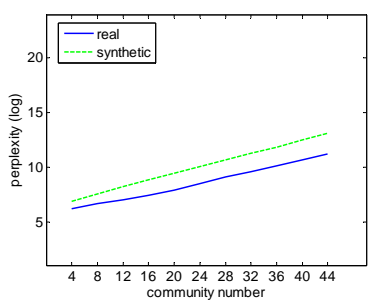

(c)

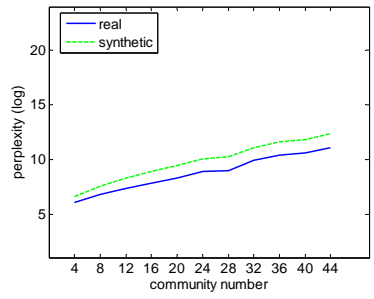

(b)

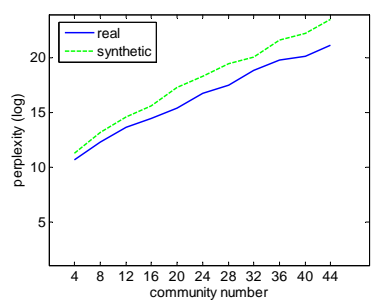

(d)
Figure 6: Perplexity for FCs for year 2007 (a)and (c) and 2008 (b) and (d). (a) and (b) correspond to FI-Comm and (c) and (d) to Role-FI-Comm. 
to FI-Comm and (c) and (d) to Role-FI-Comm. Figures $5(\mathrm{a})$ and (c) show perplexity and Figure 5 (b) and (d) show normalized perplexity.

In Figure 5(a), the perplexity of FI-Comm drops with an increasing number of topics for the seen data (2002-2005), while it initially decreases and then increases for the unseen test data in years 2006 and 2007. In comparison, in Figure $5(\mathrm{c})$, the perplexity of Role-FI-Comm decreases for the seen data (2002-2005) and increases for the unseen testing data from 2006 to 2008. From these scores, it appears that RoleFI-Comm which is a more complicated model is more likely to overfit. For both models in Figures 5 (a) and (c), the perplexity for unseen test data (2006 and 2007) is (slightly) higher than for the seen data (2002 to 2005). The perplexity for seen data in 2002 is also slightly higher. These values indicate some change in the communities over time.

We observe that the value of normalized perplexity in Figures 5 (b) and (d) is always smaller than 1.0, i.e., the perplexity score for the real dataset is always smaller than for the synthetic dataset. This indicates that the probabilistic communities of FI-Comm and Role-FI-Comm indeed capture information (topics) compared to the simple probability distribution over the FIs or Role-FI pairs (words) that was used to generate the synthetic dataset. The smaller the normalized perplexity scores, we can infer that the topic$\mathrm{s}$ are more meaningful compared to the word distribution. Comparing Figures 5 (b) to (a), the perplexity scores for the test data is similar to the score for the seen data; this also indicates topic continuity from the seen to the unseen data.

In Figure 5 (a), (b) and (c), the perplexity for year 2008 is significantly higher than the other years; this indicates that the model is not able to generalize and fit the data from the year of the subprime crisis. One observation from the dataset is that the number of FCs reduced considerably in 2008. We also know anecdotally that many large FIs went bankrupt in 2007 and 2008. However, we note that the normalized perplexity for 2008 is comparable to 2006 and 2007 in Figure 5 (d). This is an interesting difference between the two models, FI-Comm and Role-FI-Comm. An explanation is that while some FIs may not have participated in FCs, with a corresponding impact on FI-Comm, the roles played by the FIs that continued to participate did not change substantially. Hence, Role-FI-Comm perplexity scores reflect that this model was able to generalize and fit the unseen test dataset from the 2008 dataset, the abnormal subprime crisis year. This reflects well on the power of the Role-FI-Comm model.

Figure 6 shows perplexity for the real and synthetic datasets for 2007 (a) and (c) and 2008 (b) and (d). Figure 6 (a) and (b) correspond to FI-Comm and (c) and (d) to Role-FIComm. In 2007 in Figure 6 (a), the perplexity score for the real dataset is almost unchanging with increasing number of topics; the score for the synthetic dataset increases linearly. In 2008 in Figure 6 (b), the perplexity for both the real and the synthetic dataset increases superlinearly, and the perplexity of the synthetic dataset increases faster compared to the real dataset. For Role-FI-Comm, in Figures 6 (b) and (d), the perplexity score increases superlinearly; the score for the synthetic dataset and for year 2008 show the fastest increases.

\subsection{Exemplar communities and FCs}

Figures 4 and 7 illustrative Role-FI-Comm and FI-Comm, respectively. In each figure, the upper section displays the Top K Role-FIs or FIs for four or six communities. The lower section shows two or three financial contracts (FC); color coding connects the FIs or Role-FIs in the FCs to the communities. To the right of each FC in Figure 7 is a histogram of probabilities over the 16 FI-Comm communities.

Figure 7 highlights differences in the FI-Comm communities. FI384 Wells Fargo dominates FI-Comm community \#7 while FI384 Wells Fargo, FI22 American Home Mortgage and FI285 Ocwen are equally dominant in FI-Comm community \#13. We also see that FIs that are affiliated with each other participate in the same community, e.g., FI44 Bank of America Securities LLC and FI46 Bank of America, or FI101 Countrywide Home Loan and FI102 Countrywide Securities Corporation. From Figures 4 we observe that the probabilities over the Role-FIs are generally lower for Role-FI-Comm communities compared to the probabilities over FIs for the FI-Comm communities of Figure 7, which may be caused by the difference of number of discovered communities in the two illustrative figures. We will discuss quantitative evaluation of communities in Section. 4.4. We further note that an FI or Role-FI can participate in several communities.

Finally, from Figure 7, we observe that the probability distribution over the FI-Comm communities for a seen FC from 2005 and an unseen FC from 2007 are similar, i.e., both have one dominant community and another less dominant community. However, the FC from 2008 is different; it appears to be a mix over at least four FI-Comm communities. We also observe that the Top FIs from the dominant communities do not appear in the 2008 FC. For example, FI46 in dominant in community \#3 and FI384 is dominant in community \#13. While these two FIs do appear in the 2008 FC, these FIs are associated with different communities, e.g., FI384 in the 2008 FC is associated with community \#7. This reflects that the structore of the FI-Comm communities have evolved in the 2008 crisis year.

\subsection{Coherence metric of communities}

In this section, we will provide a quantitative evaluation for the discovered probabilistic communities. To evaluate the discovered communities without groundtruth is a nontrivial task. In topic modeling of text corpus, the evaluation often requires a lot of human efforts. For the resMBS data in financial domain, the evaluation would be more difficult since the financial communities are difficult to validate for humans without strong financial knowledge.

Automatic coherence detection [8] was developed to evaluate topic quality as an alternative to manual evaluation. For each community, a coherence score is calculated as the average value of pointwise mutual information (PMI) over the top $\mathrm{N}$ words of the topic. Comm-PMI is defined as follows:

$$
\text { Comm-PMI }=\sum_{j=2}^{N} \sum_{i=1}^{j-1} \log \frac{P\left(w_{j}, w_{i}\right)}{P\left(w_{i}\right) P\left(w_{j}\right)},
$$

where $w_{i}, i=1, \ldots, N$ represent the top $\mathrm{N}$ words (FIs or Role-FIs) in a community.

Table 1 reports on the coherence for FI-Comm and RoleFI-Comm with sixteen communities. The value of CommPMI is computed over the test dataset of 2006 and 2007. Based on coherence, the Role-FI-Comm communities are more interpretable compared to FI-Comm. 


\begin{tabular}{|c|c|c|c|}
\hline \multicolumn{4}{|c|}{ Probabilistic communities discovered by LDA in FI-Comm } \\
\hline Comm \#3: & & Comm \#7: & \\
\hline FI46(Bank of America) & 0.4544 & FI384(Wells Fargo) & 0.5628 \\
\hline FI44(Banc of America Securities LLC) & 0.2547 & FI363(U.S. Bank) & 0.0741 \\
\hline FI384(Wells Fargo) & 0.1186 & FI180(GreenPoint) & 0.0631 \\
\hline FI72(Cendant Mortgage) & 0.0788 & FI263(National City) & 0.0501 \\
\hline FI32(Asset Backed Funding) & 0.0498 & FI183(HSBC) & 0.0423 \\
\hline Comm \#10: & & Comm \#13: & \\
\hline FI101(Countrywide Home Loans) & 0.4754 & FI384(Wells Fargo) & 0.1856 \\
\hline FI102(Countrywide Securities Corporation) & 0.4015 & FI22(American Home Mortgage) & 0.1727 \\
\hline FI47(Bank of New York Mellon Corp.) & 0.1004 & FI285(Ocwen) & 0.1704 \\
\hline FI388(Wilmington Trust) & 0.0103 & FI364(UBS) & 0.0910 \\
\hline FI211(J.P. Morgan Chase) & 0.0071 & FI314(Renaissance Mortgage Acceptance) & 0.0830 \\
\hline Comm \#14: & & Comm \#15: & \\
\hline FI223(Lehman Brothers) & 0.2915 & FI278(Nomura Securities International Inc.) & 0.2646 \\
\hline FI34(Aurora Loan Services LLC) & 0.2808 & FI84(Citigroup) & 0.2432 \\
\hline FI344(Structured Asset) & 0.1852 & FI82(CitiMortgage) & 0.2402 \\
\hline FI384(Wells Fargo) & 0.0568 & FI171(GMAC) & 0.0993 \\
\hline FI291(Option One Mortgage Corporation) & 0.0261 & FI384(Wells Fargo) & 0.0412 \\
\hline
\end{tabular}

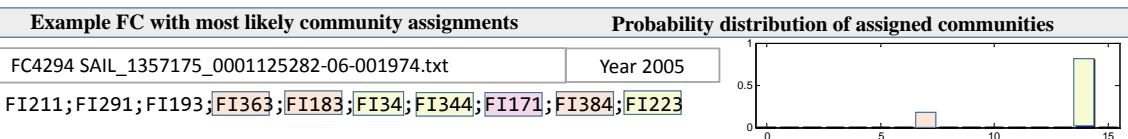

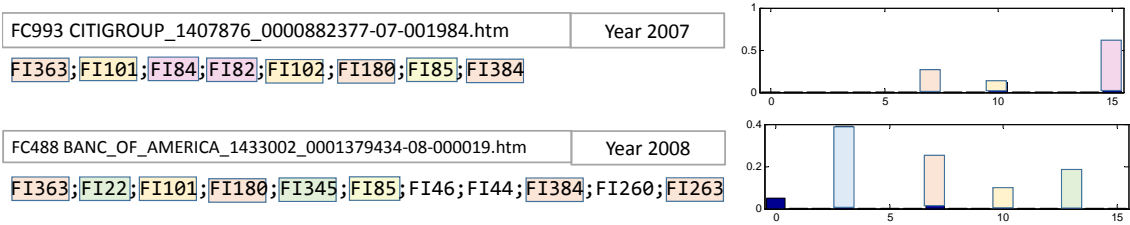

Figure 7: Financial communities (topics) from FI-Comm. The top section shows the Top FIs for six of 16 FI-Comm communities. The lower section shows three financial contracts (FC); color coding connects the FIs in the FCs to the topics. To the right of each FC is a histogram of probabilities over the 16 FI-Comm communities (topics) for each FC. The figure is best viewed in color.

\begin{tabular}{|c||c|c|c|}
\hline Comm-PMI & Top 3 & Top 5 & Top 10 \\
\hline \hline FI-Comm & 0.339 & 0.226 & 0.128 \\
\hline Role-FI-Comm & 0.491 & 0.399 & 0.357 \\
\hline
\end{tabular}

Table 1: Comm-MPI for the Top 3, 5, and 10 FIs or Role-FIs from 16 communities in FI-Comm and Role-FI-Comm.

\section{CONCLUSION AND FUTURE WORK}

In this paper, we adapted the Latent Dirichlet Allocation and developed two financial community models, FI-Comm and Role-FI-Comm. FI-Comm discovered communities of FIs and Role-FI-Comm discovered communities of Role-FI pairs. An experimental evaluation over $5000+$ financial contracts showed that both models can discover meaningful communities. In future work, we will study the temporal evolution of the FI and Role-FI communities. We will also develop graphical models that consider relational features, e.g., a particular FI only plays a particular role on contracts.

\section{ACKNOWLEDGMENTS}

The authors would like to thank Weiwei Yang for valuable feedback on topic modeling. This research was partially supported by NSF grants CNS1305368 and DBI1147144 NIST award 70NANB15H194.

\section{REFERENCES}

[1] D. M. Blei, A. Y. Ng, and M. I. Jordan. Latent dirichlet allocation. JMLR, 2003.

[2] D. Burdick, S. De, L. Raschid, M. Shao, Z. Xu, and E. Zotkina. resMBS: Constructing a financial supply chain graph from financial prospecti. In $S I G M O D$ DSMM. ACM, 2016.

[3] D. Burdick, M. Franklin, P. Issler, R. Krishnamurthy, L. Popa, L. Raschid, R. Stanton, and N. Wallace. Data science challenges in real estate asset and capital markets. In SIGMOD DSMM. ACM, 2014.

[4] D. Burdick, M. A. Hernández, H. Ho, G. Koutrika, R. Krishnamurthy, L. Popa, I. Stanoi, S. Vaithyanathan, and S. R. Das. Extracting, linking and integrating data from public sources: A financial case study. IEEE Data Eng. Bull., 2011.

[5] L. Chiticariu, R. Krishnamurthy, Y. Li, S. Raghavan, F. R. Reiss, and S. Vaithyanathan. SystemT: an algebraic approach to declarative information extraction. In $A C L, 2010$.

[6] L. Chiticariu, Y. Li, and F. R. Reiss. Rule-based information extraction is dead! long live rule-based information extraction systems! In EMNLP, 2013.

[7] M. A. Hernández, H. Ho, G. Koutrika, R. Krishnamurthy, L. Popa, I. R. Stanoi, S. Vaithyanathan, and S. Das. Unleashing the power of public data for financial risk measurement, regulation, and governance. $W W W, 2010$.

[8] J. H. Lau, D. Newman, and T. Baldwin. Machine reading tea leaves: Automatically evaluating topic coherence and topic model quality. In $E A C L, 2014$.

[9] Z. Xu, D. Burdick, and L. Raschid. Exploiting lists of names for named entity identification of financial institutions from unstructured documents. arXiv preprint arXiv:1602.04427, 2016. 